ARTí́CULO

\title{
Pre-engorda de semillas de cultivo de la almeja taquilla, Mulinia edulis, en un sistema suspendido en el mar
}

\author{
Nursery of cultured spats of the taquilla clam, Mulinia edulis, in a suspended system in the sea \\ Doris Oliva ${ }^{1,2}$, Angela del Pilar Célis ${ }^{2}$,Alejandro Abarca ${ }^{3,4}$, Marlene Pizarro $^{2}$ y L. René Durán ${ }^{2 *}$ \\ ${ }^{1}$ Instituto de Biología, Facultad de Ciencias, Universidad de Valparaíso, Av. Gran Bretaña 1111, Playa Ancha, Valparaíso, Chile \\ ${ }^{2}$ Centro de Investigación y Gestión de Recursos Naturales, Facultad de Ciencias, Universidad de Valparaíso, Av. Gran Bretaña 1111, \\ Playa Ancha, Valparaíso, Chile \\ ${ }^{3}$ Departamento de Acuicultura, Facultad de Ciencias del Mar, Universidad Católica del Norte, Larrondo 1281, Coquimbo, Chile \\ ${ }^{4}$ Centro Tecnológico de Innovación Acuícola (AquaPacífico), Coquimbo, Chile \\ *Autor corresponsal: rene.duran@uv.cl
}

\begin{abstract}
Spats of Mulinia edulis, produced in a hatchery, were nursered for 40 days in a suspended system in the sea in Bahía Yal (Chiloé), in order to test the feasibility of pre-fattening. Two initial sizes: 2.9 and $6.2 \mathrm{~mm}$ and two cultivation densities [1(D1) and 2(D2) spats $\mathrm{cm}^{-2}$ ] were used in treatments with (TA) and without sand (TSA) with small spats. Additionally, the growth and the survival of small spats in long-line installed both on the periphery and in the center of the farm were tested. In the size experiment, the small spats grew an average of $5.0 \mathrm{~mm}$ and the large spats an average of $4.9 \mathrm{~mm}$ in TA, and in TSA, $0.9 \mathrm{~mm}$ and $0.7 \mathrm{~mm}$, respectively. The spat survival in TA (small $=51.3 \%$ and large $=62.3 \%$ ) was greater than in TSA (small= $18.9 \%$ and large $=40.0 \%$ ). In the density experiment, the largest sizes were reached in the TA, with an average growth of $5.0 \mathrm{~mm}$ in D1 and $4.5 \mathrm{~mm}$ in D2. The greatest survival was attained in TA ( $1=51.3 \%$ and $D 2=49.4 \%$ ) and the lowest in the TSA (D1= 18.9\% and D2= 7.1\%). The growth of the small spats was greater in the long-line located in the periphery of the farm, nevertheless no differences were observed in the survival. The results show that it is feasible to do large-scale nursering with an initial spat size of $2.9 \mathrm{~mm}$ at a density of 2 spat $\mathrm{cm}^{2}$ in a suspended system with sand.
\end{abstract}

Key words: Mulinia, clam, seed production, pre-fattening, long-line

Resumen.- Semillas (spats) de Mulinia edulis, producidas en hatchery, se engordaron durante 40 días, en un sistema de cultivo suspendido (long-line) en el mar en Bahía Yal (Chiloé, Chile), con el objetivo de probar la factibilidad de pre-engorda. Se utilizaron dos tamaños de siembra 2,9 y 6,2 mm y dos densidades [1 (D1) y 2 (D2) semillas $\mathrm{cm}^{-2}$ ] en tratamientos con (TA) y sin arena (TSA) con semillas pequeñas. Se determinó el crecimiento y la supervivencia de semillas pequeñas en long-line instalados en la periferia y en el centro de la concesión de acuicultura. En el experimento de tamaño de siembra las semillas pequeñas crecieron en promedio 5,0 $\mathrm{mm}$ y las grandes $4,9 \mathrm{~mm}$ en TA y en el TSA 0,9 mm y 0,7 mm, respectivamente. La supervivencia de las semillas en TA (pequeñas= $51,3 \%$; grandes= 62,3\%) fue mayor que en TSA (pequeñas=18,9\%; grandes= 40,0\%). En el experimento de densidad, las mayores longitudes se presentaron en TA, con un crecimiento promedio 5,0 mm en D1 y 4,5 mm en D2. La mayor supervivencia se obtuvo en TA (D1= 51,3\%; D2= 49,4\%) y la menor en TSA (D1= 18,9\%; D2=7,1\%. El crecimiento de las semillas pequeñas fue mayor en los long-line ubicados en la periferia, no observándose diferencias en la supervivencia. Los resultados muestran que es factible realizar una pre-engorda a un tamaño de siembra de $2,9 \mathrm{~mm}$ con una densidad inicial de 2 semillas $\mathrm{cm}^{-2}$ en sistemas suspendidos con arena.

Palabras clave: Mulinia, almeja, producción de semillas, pre-engorda, sistema suspendido

\section{INTRODUCCIÓN}

Para el desarrollo de la acuicultura de pequeña escala (APE), es necesario incorporar tecnología de cultivo para la producción de semillas de moluscos enterradores. En Chile, en un estudio elaborado por CORFO (2015) se sugiere potenciar la investigación en cinco especies de bivalvos enterradores, Ameghinomya antiqua (King, 1832), Mulinia edulis (King \& Broderip, 1832), Gari solida (Gray, 1828), Mesodesma donacium (Lamarck, 1818) y Ensis macha (Molina, 1782).
La almeja taquilla, M. edulis, se distribuye desde Callao, Perú, hasta el Estrecho de Magallanes y Tierra del Fuego en Chile (Oliva et al. 2005, Jaramillo et al. 2008). Habita en la zona intermareal inferior y submareal somero en fondos de arena fina entre 2 y $17 \mathrm{~m}$ de profundidad; presenta un ciclo reproductivo continuo y es una especie fácil de manipular en el laboratorio (Oliva et al. 2005, Stotz et al. 2008). 
La tecnología de cultivo larvario para la almeja taquilla se ha desarrollado en forma experimental y se ha escalado a producciones piloto en estanques de $1000 \mathrm{~L}$ (Oliva et al. 2014, Vivanco et al. 2014). El cultivo de las postlarvas y semillas (spat) se ha realizado en sistemas down-welling y también en sistemas cerrados con aireación y con una capa de arena (Oliva et al. 2013, 2014) obteniendo semillas que han sido engordadas con éxito en sistemas de fondo, en la zona norte y sur de Chile (Abarca et al. 2012).

El traslado de las semillas desde el hatchery hacia el medio natural debe realizarse tempranamente, con el objeto de abaratar los costos asociados a la alimentación y liberar espacio en el hatchery para la siguiente cohorte de larvas pedivelígeras (Jones et al. 1993, Hadley et al. 1997, da Costa et al. 2011, 2012).

A pesar de que el cultivo de almejas ha alcanzado un alto nivel de desarrollo en América del Norte y Europa (Manzi \& Castagna 1989, da Costa 2012), el ingreso de semillas al ambiente natural presenta altas mortalidades a tamaños menores a $10 \mathrm{~mm}$. En el caso de Mercenaria mercenaria (Eldridge et al. 1979, Manzi et al. 1986), Tapes philippinarum (Jones et al. 1993) y Ensis arcuatus, se necesita una fase intermedia de cultivo, en un sistema protegido, antes de ser transferidos directamente a los sistemas de engorda (da Costa et al. 2015). Varias soluciones se han propuesto para implementar sistemas intermedios de cultivo: sistemas de raceway en tierra, estanques con upwelling o sistemas de upwelling flotantes en el mar (Hadley et al. 1997, Castagna 2001).

En Chile se han desarrollado los sistemas de cultivo suspendidos para la acuicultura de bivalvos utilizando sistemas de long-line simple principalmente para el cultivo de pectínidos (von Brand et al. 2016) y long-line doble para el cultivo de mitílidos (Uriarte 2008). Estos sistemas de crecimiento mantienen a los bivalvos en la columna de agua para que puedan alimentarse naturalmente hasta alcanzar el tamaño comercial.

El objetivo de este trabajo fue probar la factibilidad de realizar una pre-engorda de semillas de 60 y 90 días de edad para la almeja taquilla en sistema suspendidos en la columna de agua con y sin arena. El estudio se realizó en un centro de mitilicultura, comparando el crecimiento y la supervivencia de semillas de dos tamaños de siembra, en sistemas con y sin arena y dos densidades de cultivo. Además, se evaluó el efecto de la ubicación espacial, en el centro o en la periferia de la concesión de acuicultura, de las unidades experimentales.

\section{MATERIALES Y METODOS}

\section{Producción de SEMILlas}

Se cultivaron larvas de la almeja taquilla, Mulinia edulis en estanques de 1000 L en el hatchery de Orizon S.A. ubicado en la localidad de Teupa (Región de los Lagos, Chiloé, $\left.42^{\circ} 29^{\prime} \mathrm{S}, 73^{\circ} 32^{\prime} \mathrm{O}\right)$. Los reproductores fueron recolectados en Quetalmahue, Chiloe ( $49^{\circ} 51^{\prime}$ 'S, $\left.73^{\circ} 58^{\prime} \mathrm{O}\right)$ y se realizaron desoves, inducidos por cambios de temperatura, en el invierno de 2012. Las larvas velígeras se cultivaron en agua de mar filtrada a $5 \mu \mathrm{m}$, esterilizada con luz UV y a una temperatura de $15 \pm 1{ }^{\circ} \mathrm{C}$. Inicialmente, se alimentaron con Isochrysis aff. galbana (Clon T-ISO) a una concentración de $25.000 \mathrm{cél} \cdot \mathrm{ml}^{-1} \cdot \mathrm{día}^{-1}$. Al cabo de 10 días se aumentó la ración a $50.000 \mathrm{cél} \cdot \mathrm{ml}^{-1} \cdot$ día $^{-1}$, agregando la diatomea Chaetoceros gracilis en una relación 1:1 hasta alcanzar una longitud larval de 280-290 $\mu \mathrm{m}$ previo a su asentamiento (Oliva et al. 2014, Vivanco et al. 2014).

Las larvas se asentaron en un sistema downwelling con malla de $150 \mu \mathrm{m}$ y se mantuvieron en el hatchery durante 60 días las pequeñas $(2,9 \pm 0,7 \mathrm{~mm})$ y durante 90 días las grandes $(6,2 \pm 0,7 \mathrm{~mm})$. La ración de alimento se aumentó en las 4 primeras semanas de 50.000 a 100.000 cél $\cdot \mathrm{ml}^{-1} \cdot$ día $^{-1}$.

\section{DISEÑO EXPERIMENTAL}

Durante la primavera de 2012 (septiembre) se instalaron en forma simultánea en la concesión de mitilicultura de Orizon S.A. tres experimentos: a) dos tamaños de siembra con y sin arena, b) dos densidades de siembra con y sin arena para semillas pequeñas $\mathrm{y}, \mathrm{c}$ ) dos ubicaciones en la periferia versus en el centro de la concesión para semillas pequeñas (Figs. 1 y 2). Todos los tratamientos en cada uno de los experimentos se instalaron en triplicado. En un long-line de cultivo de mejillones se colgaron canastos de plástico rígido de base circular de $254 \mathrm{~cm}^{2}$ a una profundidad de $5 \mathrm{~m}$. La base y los primeros $7 \mathrm{~cm}$ inferiores de los canastos eran sólidos para evitar la pérdida de arena. En los tratamientos con arena (TA) se adicionó una capa de $3 \mathrm{~cm}$ de arena fina (entre 63 y $125 \mu \mathrm{m}$ ). Los canastos se cubrieron externamente con una malla plástica de $1 \mathrm{~mm}$ de abertura la que se unió mediante un cabo a la línea madre del long-line. En su parte inferior se instaló un peso para estabilizar los canastos. En los tratamientos sin arena (TSA), las semillas se instalaron directamente en el fondo de los canastos. Basado en los antecedentes de Cerviño (2011) para Venerupis pullastra, y de Oliva et al. (2005) para M. edulis, los experimentos se mantuvieron 40 días suspendidos en el long-line en el mar y al cabo de este tiempo se retiraron y las semillas fueron medidas y contadas, para determinar su crecimiento (longitud valvar en $\mathrm{mm}$ ) y supervivencia (\%). 


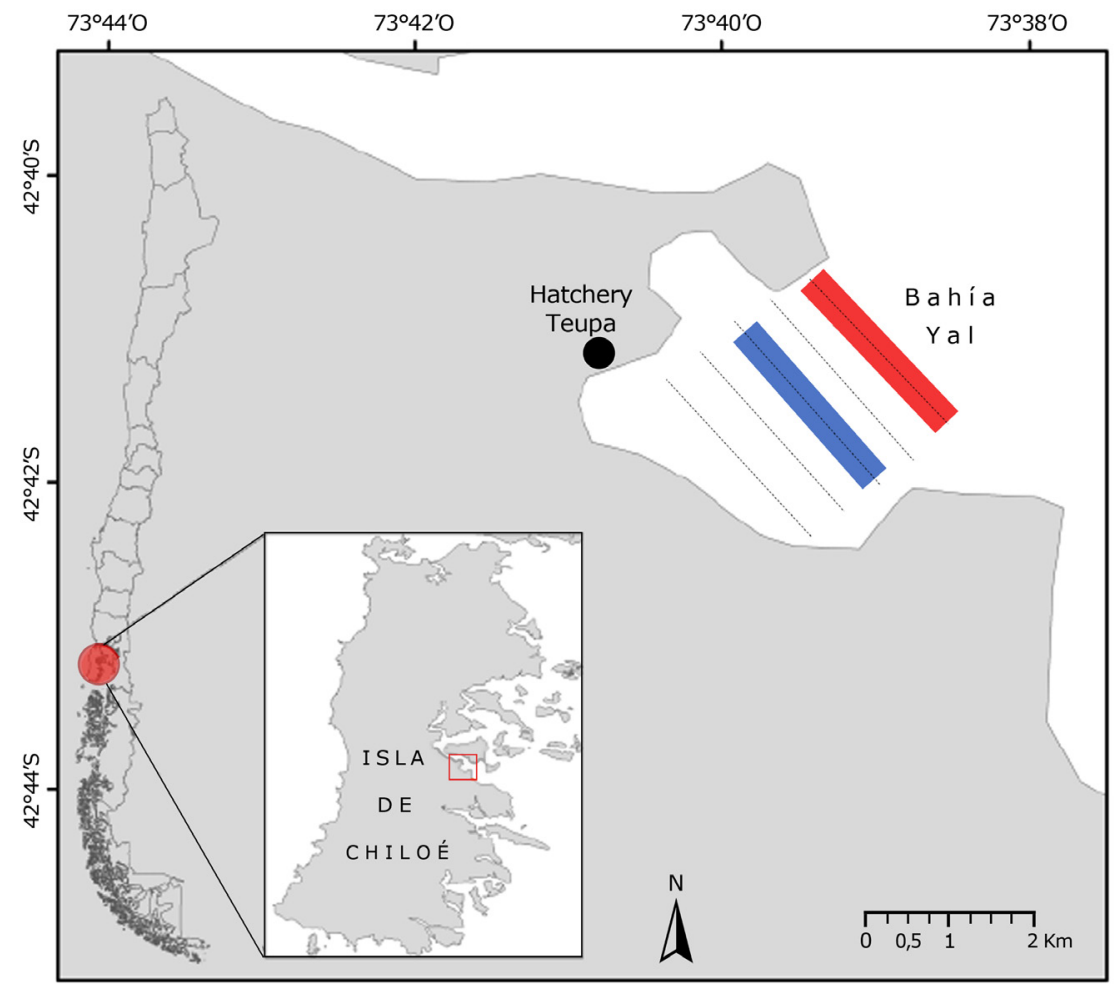

Figura 1. Ubicación de las zonas de instalación de los experimentos de pre-engorda de semillas de Mulinia edulis en la concesión de Orizon S.A. en sector de Teupa $\left(42^{\circ} 29^{\prime} \mathrm{S} ; 73^{\circ} 32^{\prime} \mathrm{O}\right)$. El rectángulo rojo corresponde a long-line ubicado en la periferia y el azul en el centro de la concesión de acuicultura / Location of the experiments of the pre-fattening of Mulinia edulis spats at the Orizon S.A. farm, in Teupa $\left(42^{\circ} 29^{\prime} \mathrm{S} ; 73^{\circ} 32^{\prime} \mathrm{W}\right)$. Red rectangle indicates long-line located in the periphery and blue rectangle in the center of the farm

\begin{tabular}{|c|c|c|c|c|c|c|c|}
\hline \multicolumn{2}{|c|}{ Tamaño siembra } & $2,9 \mathrm{~mm}$ & $2,9 \mathrm{~mm}$ & $2,9 \mathrm{~mm}$ & $2,9 \mathrm{~mm}$ & \multirow{2}{*}{$\begin{array}{l}6,2 \mathrm{~mm} \\
\text { Sin Arena }\end{array}$} & $6,2 \mathrm{~mm}$ \\
\hline & Sustrato & Sin Arena & Con Arena & Sin Arena & Con Arena & & Con Arena \\
\hline & Densidad & D2 & D2 & D1 & D1 & D1 & D1 \\
\hline \multirow{2}{*}{\multicolumn{2}{|c|}{ Ubicación }} & Centro & Centro & Centro & Centro & Centro & Centro \\
\hline & & & & & $2,9 \mathrm{~mm}$ & i & \\
\hline A & - & \multicolumn{2}{|c|}{ Tamaño siembra y sustrato } & & Con Arena & i & \\
\hline B & "..." & Densidad y S & strato & & D1 & $i$ & \\
\hline $\mathrm{C}$ &.---- & Ubicación en & concesión & & Periferia & i & \\
\hline
\end{tabular}

Figura 2. Diseño experimental para evaluar el crecimiento y supervivencia de semillas de Mulinia edulis en experimentos con A) dos tamaños de siembra con y sin arena, B) dos densidades de cultivo para semillas pequeñas con y sin arena (D1= densidad de $1 \mathrm{semilla}^{\mathrm{cm}}{ }^{-2}$, D2= densidad de 2 semilla $\mathrm{cm}^{-2}$ ) y C) dos ubicaciones (periferia y centro de la concesión de acuicultura) / Experimental design to evaluate growth and survival of Mulinia edulis spats in experiments A) two planting sizes with and without sand, B) two culture densities for small spats with and without sand (D1= density of 1 spat $\mathrm{cm}^{-2}, \mathrm{D} 2=$ density of 2 spat $\mathrm{cm}^{-2}$ ) and C) two locations (periphery and center of the farm) 
Pre-engorda con Semillas de dos tamaños de SIEMBRA CON Y SIN ARENA

Se ensayaron dos tamaños de siembra para las semillas: a) pequeñas y b) grandes las que se sembraron a una densidad de 1 semilla $\mathrm{cm}^{-2}$ (D1) (Fig. 2).

\section{Pre-ENgorda de SEMILlas CON DOS DENSIDAdeS DE} SIEMBRA CON Y SIN ARENA

Con las semillas pequeñas se realizó un segundo experimento para determinar si la densidad afecta el crecimiento y la supervivencia, en sistemas con y sin arena (Fig. 2). La densidad de cultivo usada fue 1 semilla $\mathrm{cm}^{-2}$ (D1) y 2 semillas $\mathrm{cm}^{-2}$ (D2) de acuerdo a los sugerido por Oliva et al. (2005).

En ambos experimentos (tamaño de siembra y densidad), los sistemas suspendidos fueron colocados en un long-line ubicado al centro de la concesión de mejillones (Fig. 1).

\section{Pre-engorda de semillas ubicadas en la PERIFERIA Y CENTRO DE LA CONCESIÓN}

Para esta experiencia, se instalaron tres réplicas del TA sembradas con semillas pequeñas a una densidad D1 en un long-line ubicado en la periferia de la concesion de acuicultura (Figs. 1, 2). Se comparó el crecimiento y la supervivencia de estas semillas con aquellas ubicadas en el centro del cultivo.

\section{ANALISIS ESTADistico}

Previo a los análisis estadísticos, se revisaron los supuestos de normalidad y homogeneidad de varianza, utilizando las pruebas de Shapiro-Wilk y Levene, respectivamente. En caso de ser necesario, se realizaron transformaciones logarítmicas para satisfacer los supuestos mencionados. Para la evaluación de los porcentajes de supervivencia en cada tratamiento, los datos fueron previamente transformados a valor arcoseno de la raíz de la proporción del porcentaje. Para comparar el efecto de la presencia de sustrato (TA $v / s$ TSA) sobre el crecimiento y la supervivencia de las semillas grandes y el efecto de la ubicación de la línea de cultivo en la concesión sobre el crecimiento y la supervivencia de las semillas se realizó una prueba t-Student, previa prueba $\mathrm{F}$ para varianza de dos muestras, con el objeto de verificar homogeneidad de varianzas. Se utilizó un ANOVA de dos vías para evaluar el efecto de la densidad de cultivo y el sustrato sobre el crecimiento y la supervivencia de las semillas pequeñas (Sokal \& Rohlf 2012). Para todos los análisis se utilizó el software Statistica 12.0 (StatSoft, Inc).

\section{RESUltados}

Pre-engorda con Semillas de dos tamaños de SIEMBRA CON Y SIN ARENA

En el tratamiento con arena (TA), tanto para las semillas pequeñas $(\mathrm{t}=23,75 ; \mathrm{g} .1=137 ; P<0,001)$ como grandes $(\mathrm{t}=$ $12,82 ;$ g.l $=118 ; P<0,001)$ se obtuvo un incremento en la longitud valvar significamente mayor que en el tratamiento sin arena (TSA) (Fig. 3A). Las semillas pequeñas $(2,9 \pm$ $0,7 \mathrm{~mm}$ ) obtuvieron una longitud final de 7,9 $\pm 1,4 \mathrm{~mm}$, creciendo en promedio 5,0 mm en 40 días. Las semillas grandes $(6,2 \pm 0,70 \mathrm{~mm})$ alcanzaron una longitud promedio de $11,1 \pm 2,9 \mathrm{~mm}$, creciendo 4,9 $\mathrm{mm}$ en el mismo periodo. En el TSA las semillas pequeñas crecieron hasta un tamaño de 3,8 $\pm 0,7 \mathrm{~mm}$, es decir $0.9 \mathrm{~mm}$ en 40 días (Fig. 3A) y en el caso de las semillas grandes la longitud promedio en el periodo experimental alcanzó $6,9 \pm 1,2 \mathrm{~mm}$, con un crecimiento de $0,7 \mathrm{~mm}$.

A
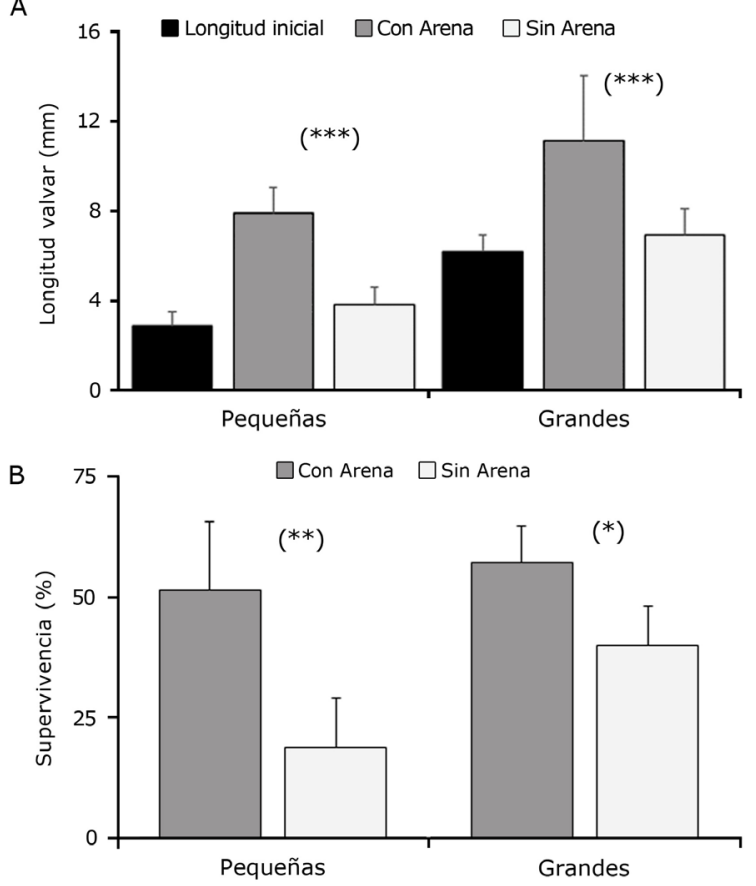

Figura 3. Longitud valvar ( $\mathrm{mm}$ ) inicial y final (A) y supervivencia (\%) (B) de semillas pequeñas y grandes de Mulinia edulis (promedio \pm d.e.) mantenidas en sistemas suspendidos en tratamientos con y sin arena $\left({ }^{*}\right): P<0,05 ;\left({ }^{* *}\right): P<0,01 ;(* * *): P<0,001 /$ Initial and final shell length $(\mathrm{mm})(A)$ and survival (\%) (B) of small and large spats of the Mulinia edulis (mean \pm s.d.) maintained in suspended systems in treatments with and without sand 
La supervivencia fue mayor en los TA que en los TSA para ambos tamaños de siembra de semillas de $M$. edulis (Fig. 3B). En TA, la supervivencia en las semillas pequeñas fue de $51,3 \pm 14,2 \%$ y en las grandes de $62,3 \pm$ $5,4 \%$ y en TSA la supervivencia de las semillas pequeñas fue de $18,9 \pm 10,1 \%$ y las grandes presentaron una mayor supervivencia con un 40,0 $\pm 8,1 \%$. Una comparación de $\mathrm{t}$ de Student entre los valores de supervivencia en TA y TSA mostró diferencias significativas tanto para las semillas pequeñas $(\mathrm{t}=3,95 ;$ g.l. $=4 ; P=0,008)$ como para las semillas grandes $(\mathrm{t}=3,22 ; \mathrm{g} .1 .=4 ; P=0,016)$.

\section{Pre-engorda de SEMILLAS CON DOS DENSIDAdeS DE SIEMBRA CON Y SIN ARENA}

Las semillas pequeñas cultivadas en el mar a una densidad de 1 semilla cm-2 (D1) con arena (TA) alcanzaron la mayor longitud valvar con 7,9 $\pm 1,4 \mathrm{~mm}$ y aquellas cultivadas a una densidad de 2 semillas $\mathrm{cm}^{-2}$ (D2) alcanzaron un tamaño promedio de 7,4 $\pm 1,5 \mathrm{~mm}$, no detectándose diferencias significativas en el crecimiento entre ambas densidades $(\mathrm{F}=0,22 ;$ g.1. $=1 ; P=0,64$; Tabla 1, Fig. 4A).

A

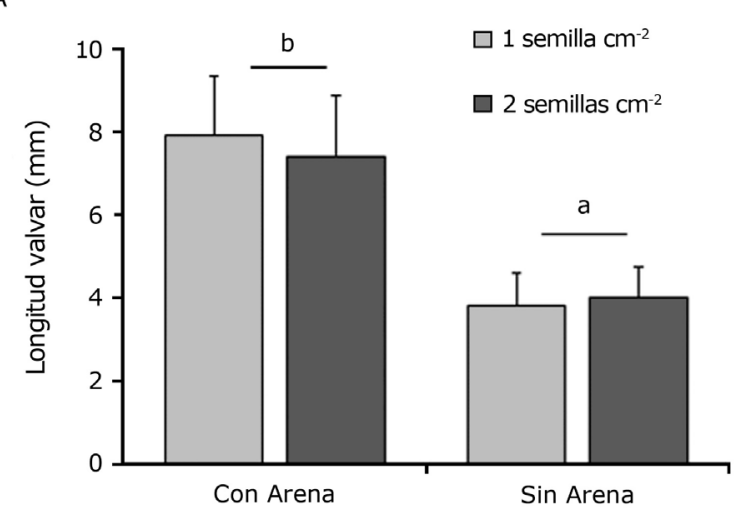

B

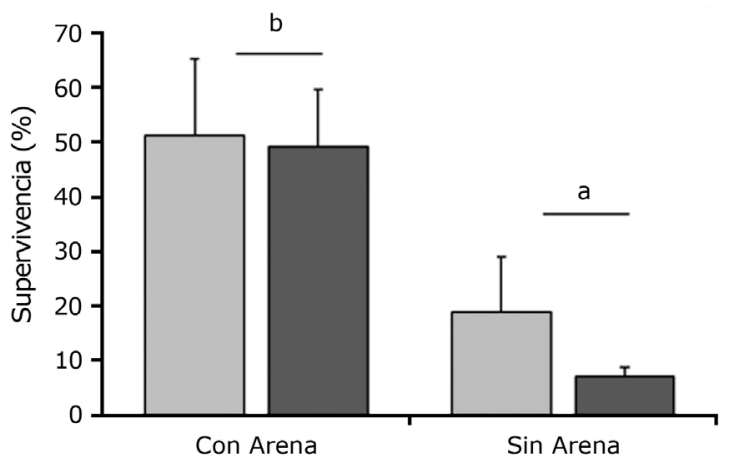

Figura 4. Longitud valvar ( $\mathrm{mm}$ ) (A) y supervivencia (\%) (B) de semillas pequeñas de Mulinia edulis (promedio \pm d.e.) cultivadas con dos densidades y mantenidas en sistemas suspendidos en tratamientos con y sin arena / Shell length $(\mathrm{mm})(A)$ and survival (\%) (B) of small spats of Mulinia edulis (mean \pm s.d.) cultivated at two densities and maintained in suspended systems in treatments with and without sand
Las semillas engordadas en el sistema en TSA a densidad D1 alcanzaron un tamaño final de 3,8 $\pm 0,8 \mathrm{~mm}$, en cambio, las semillas mantenidas al doble de la densidad (D2) lograron un tamaño final promedio ligeramente mayor con 4,0 $\pm 0,7 \mathrm{~mm}$ (Fig. 4A).

El análisis de varianza de dos vías evidenció que las semillas engordadas en arena presentaron un crecimiento significativamente mayor $(\mathrm{F}=1084,52 ;$ g.1. $=1 ; P<0,001)$ que aquellas sin arena (Tabla 1$)$.

La densidad no afectó significativamente la supervivencia $(\mathrm{F}=1,34 ;$ g.l. $=1 ; P=0,28$; Tabla 1$)$, sin embargo, en presencia de arena la supervivencia fue significativamente mayor $(\mathrm{F}=40,00 ;$ g.l. $=1 ; P<0,001)$ (Fig. 4B). La mayor supervivencia se obtuvo en el TA con un $51,3 \pm 14,2 \%$ para las semillas mantenidas en D1 y de un 49,4 $\pm 10,5 \%$ para las semillas a una densidad D2. El menor valor de supervivencia $(7,1 \pm 1,7 \%)$ se registró en el TSA con densidad D2. A la densidad menor (D1), la supervivencia alcanzó un $18,9 \pm 10,1 \%$.

Tabla 1. Análisis de varianza de dos vías para evaluar el efecto de la densidad (1 [D1] y 2 [D2] semillas $\mathrm{cm}^{-2}$ ) y el sustrato (con [TA] y sin arena [TSA]) y sobre el crecimiento en la longitud de la valva $(\mathrm{mm})$ y la supervivencia en semillas pequeñas de la almeja Mulinia edulis / Two way analysis of variance to evaluate the effect of the spat density (1 [D1] and 2 [D2] spats $\mathrm{cm}^{-2}$ ) and the substrate (with [TA] and without [TSA] sand) on the growth in shell length $(\mathrm{mm})$ and survival in spats of the Mulinia edulis clam

\begin{tabular}{lcccl}
\hline & g.l & MS & F & $P$ \\
\hline Crecimiento & & & & \\
Densidad (D1-D2) & 1 & 0,002 & 0,22 & 0,640 \\
Sustrato (TA-TSA) & 1 & 7,648 & 1084,52 & $0,000^{*}$ \\
Densidad* Sustrato & 1 & 0,060 & 8,51 & $0,004^{*}$ \\
Dentro del grupo & 356 & 0,007 & & \\
Total & 359 & & & \\
Supervivencia & & & & \\
Densidad (D1-D2) & 1 & 139,707 & 1,34 & 0,281 \\
Sustrato (TA-TSA) & 1 & 4181,921 & 40,00 & $0,000^{*}$ \\
Densidad* Sustrato & 1 & 72,656 & 0,69 & 0,429 \\
Dentro del grupo & 8 & 104,535 & & \\
Total & 11 & & & \\
\hline
\end{tabular}

* indican diferencias significativas 


\section{Pre-engorda de semillas ubicadas en la PERIFERIA Y CENTRO DE LA CONCESIÓN}

Al comparar el crecimiento y la supervivencia en función de la ubicación de los ejemplares dentro de la concesión en TA (Fig. 1), se evidenció que el crecimiento de las semillas en la periferia fue significativamente mayor $(8,3$ $\pm 1,8 \mathrm{~mm}$ ) respecto de los ejemplares sembrados en el centro de la concesión $(7,9 \pm 2,1 \mathrm{~mm})(\mathrm{t}=1,75 ; \mathrm{g} . \mathrm{l}=178$; $P=0,04)$. No se encontraron diferencias significativas en la supervivencia entre ambos grupos con $53,9 \pm 12,7 \%$ y $51,3 \pm 14,2 \%$ y respectivamente $(\mathrm{t}=0,24 ; \mathrm{g} \cdot 1 .=4 ; P=0,41)$.

\section{DisCUSIÓN}

En el presente estudio, se determinó que los cultivos suspendidos con arena permiten un mayor crecimiento y una mayor supervivencia en las semillas de la almeja Mulinia edulis.

En condiciones de hatchery, el desarrollo de las semillas está determinada por la densidad de cultivo, la calidad del agua y del alimento a suministrar (Oliva et al. 2014). A medida que las postlarvas crecen, aumenta la cantidad de alimento requerido (Epelbaum et al. 2011, Liu et al. 2011, Oliva et al. 2013). La competencia por alimento entre las larvas en cultivo, el grado de confinamiento que redunda en un deterioro en la calidad del agua (Raghavan \& Gopinathan 2008) y el alto costo de la alimentación en esta etapa (Hadley et al. 1997, da Costa et al. 2011, Oostlander et al. 2020) hacen necesario trasladar las semillas al medio ambiente con arena y en la etapa más temprana posible en su desarrollo.

La siembra en el ambiente natural de semillas de Mercenaria mercenaria menores a $8 \mathrm{~mm}$ de longitud genera pérdidas por depredación, incluso utilizando mallas de exclusión. Con semillas sembradas entre los $15-20 \mathrm{~mm}$ se logra una producción exitosa en sistemas extensivos intermareales y submareales (Eldridge et al. 1979, Castagna 1984, 2001). En el caso de M. edulis se ha evidenciado un buen crecimiento con semillas sembradas en sistemas de fondo con arena y tamaños iniciales desde 7,5 mm de longitud (Abarca et al. 2012)

Los sistemas de nursery han sido ampliamente utilizados en Norteamérica para los cultivos de almejas, algunos en instalaciones en tierra como es el caso de los raceways (Castagna 1984, 2001) o directamente en el mar con sistemas de flujo ascendente (Manzi et al. 1986, Hadley et al. 1997, Castagna 2001). En el presente trabajo se planteó realizar esta etapa intermedia entre hatchery y sistemas de fondo, usando sistemas suspendidos en long-line de cultivo de mitílidos. Los resultados indican que la talla de siembra en sistemas suspendidos en el mar, en esta especie, puede comenzar desde tamaños cercanos a los $3 \mathrm{~mm}$ de longitud, no existiendo diferencias en la supervivencia con semillas ingresadas a un tamaño mayor ( $>6 \mathrm{~mm}$ ). De esta forma se optimizan los recursos en el hatchery al sacar las semillas tempranamente del sistema controlado y permitir que se puedan desarrollar y crecer en el mar en un periodo corto de tiempo (40 días), alcanzando así los 7,5 mm para llevarlos a los sistemas de fondo (Abarca et al. 2012).

El manejo de la arena en sistemas de cultivo suspendido desde el punto de vista operacional es complejo ya que aumenta el peso y dificulta la manipulación de los sistemas. Sin embargo, esto se compensa con los mayores valores de supervivencia que se obtienen, en este caso, un $51 \%$ en los sistemas con arena versus $19 \%$ en los sistemas sin arena para las semillas pequeñas y $62 \%$ versus $40 \%$ para las semillas grandes. Semillas de otra cohorte de $M$. edulis mantenidas en el laboratorio por 30 días crecieron de 2,8 a 4,2 mm, siendo el crecimiento menor al obtenido en el ambiente natural, pero con una alta supervivencia (75\%) (Gutiérrez et al. 2001) ${ }^{1}$. Por otro lado, experiencias realizadas por Royo et al. (2005) en Ruditapes philippinarum han demostrado que esta almeja puede crecer en sistemas suspendidos sin arena desde un tamaño de siembra cercano a 4,5 mm; sin embargo, un $38 \%$ de estas semillas de almeja sufrieron deformaciones en las valvas. En M. edulis en los experimentos sin arena no se observaron deformaciones en la concha, pero el crecimiento fue inferior al de los sistemas con arena. Estudios realizados en Clinocardium nuttallii (Epelbaum et al. 2011, Dunham et al. 2013) comparando sistemas de cultivo en el intermareal y suspendidos demostraron que el sistema suspendido con arena generó una supervivencia alta $(>96 \%)$ en las diferentes densidades de siembra probadas.

La densidad de cultivo en sistemas suspendidos no afectó el crecimiento ni la supervivencia (Tabla 1) en semillas pequeñas de 2,9 mm. Esta información indica que las semillas pequeñas son capaces de doblar o triplicar su tamaño inicial alimentándose de forma natural y obteniendo supervivencias sobre el $50 \%$ en 40 días. Por otro lado, se debe tomar en cuenta que la experiencia de cultivo se realizó en septiembre, con temperatura superficial del mar entre 9 y $12{ }^{\circ} \mathrm{C}$ por lo que sería esperable aún mejores resultados en condiciones de verano (enerofebrero) con temperaturas entre 12 y $16^{\circ} \mathrm{C}$. Los resultados

${ }^{1}$ Gutiérrez R, L Herrera, A Abarca \& D Oliva. 2001. Primera producción masiva de larvas y semillas de la almeja taquilla Mulinia edulis. XXI Congreso del Ciencias del Mar, Viña del Mar, p. 95. [resumen] 
de crecimiento obtenidos para M. edulis son equivalentes a los de Cerviño (2011) en Venerupis pullastra en cultivos de 38 días con tallas de siembra de 3,9 mm, sin embargo, la supervivencia que obtuvieron fue superior (95\%) a la obtenida para M. edulis.

En resumen, el cultivo en sistemas suspendidos en presencia de sustrato (arena) con semillas de M edulis, de 2,9 $\mathrm{mm}$ a una densidad de 2 semillas $\mathrm{cm}^{-2}$ es exitoso. Sin embargo, es importante tener en cuenta la ubicación para la instalación de los sistemas de cultivo en la concesión de acuicultura. En la presente experiencia, los ejemplares colocados en la periferia, donde hay mayor disponibilidad de alimento, presentaron los resultados de crecimiento mayores.

Los resultados de crecimiento y supervivencia de estos experimentos de pre-engorda permiten aportar una nueva pieza de información en el proceso de cultivo de la almeja taquilla. Partiendo con 10 millones de larvas $(93 \mu \mathrm{m})$ en estanques de 1000 L se obtienen en 14-16 días larvas pedivelígeras $(223 \mu \mathrm{m})$ con un $44 \%$ de supervivencia. Las semillas tempranas $(2.370 \mu \mathrm{m})$ se cultivan en un sistema downwelling por 35-45 días hasta los $2 \mathrm{~mm}$ con una supervivencia de 68\% (Oliva et al. 2014). Luego, las semillas tempranas se trasladan a un sistema intermedio de pre-engorda o nursery en sistema de cultivo suspendido en el mar, con una capa de arena fina y se logra en 40 días una talla media de $7,9 \mathrm{~mm}$ y una supervivencia de $51 \%$ (este trabajo). Experiencias con semillas producidas en el hatchery de Teupa demostraron que son capaces de resistir largos períodos de transporte y enterrarse en forma exitosa (Abarca et al. 2019). Finalmente, el traslado a un sistema de fondo en la zona intermareal durante la época de primavera produce una supervivencia del $88 \%$ obteniendo almejas de pequeño calibre de $3 \mathrm{~cm}$ en 8 meses con un peso total cercano a los $4 \mathrm{~g}$ de peso húmedo (Abarca et al. 2012). De este modo, en 11 meses se pueden obtener 1,3 millones de almejas de pequeño calibre a partir de un batch con una biomasa de 5,2 t. Estas cifras que se han obtenido en tres etapas (hatchery, cultivo de fondo y cultivo suspendido) en diferentes tiempos, ahora deben ser escaladas en cultivos masivos cubriendo todo el ciclo en forma secuencial para su validación. En los cultivos suspendidos es importante avanzar en probar la estacionalidad en la siembra, utilización de densidades mayores e innovar en el diseño de sistemas.

\section{Agradecimientos}

Agradecemos el financiamiento de los proyectos FONDEF AQ08I 1027 y CORFO INNOVA 14 IDL4-30365 у а Gonzalo Fernández de Orizon S.A. por el apoyo en el desarrollo de los experimentos. Además, agradecemos a los revisores anónimos sus comentarios que permitieron mejorar el manuscrito.

\section{LiTERATURA CITADA}

Abarca A, D Oliva, R Gutiérrez, Á Celis \& R Durán. 2012. Grown-out of seeds of the taquilla clam Mulinia edulis (King \& Broderip, 1832) in the subtidal zone in northern Chile and in the intertidal zone in southern Chile. Latin American Journal of Aquatic Research 40(3): 694-704.

Abarca A, D Oliva \& P Toledo. 2019. Effect of transfer time, temperature, and size on burrowing capacity of juvenile clams, Mulinia edulis, from hatchery. World Aquaculture Society 50(4): 707-886.

Castagna M. 1984. Methods of growing Mercenaria mercenaria from postlarval to preferred-size seed for field planting. Aquaculture 39: 355-359.

Castagna M. 2001. Aquaculture of the hard clam, Mercenaria mercenaria. In: Kraeuter JN \& M Castagna (eds). Biology of the hard clam. Developments in Aquaculture and Fisheries Science 31: 675-699. Elsevier Publication, New York.

Cerviño A. 2011. Ciclo reproductivo, cultivo en criadero y en el medio natural de la almeja babosa Venerupis pullastra (Montagu, 1803). Memoria de Doctora en Biología, Instituto de Acuicultura, Universidad de Santiago de Compostela, Santiago de Compostela, 288 pp.

CORFO. 2015. Selección de nuevas especies de cultivo para la diversificación de la acuicultura en Chile, 88 pp. CORFO, Santiago.

Da Costa F. 2012. Clam fisheries and aquaculture, 379 pp. Nova Science Publishers, New York.

Da Costa F, S Darriba, D Martinez-Patiño \& A Guerra. 2011. Culture possibilities of the razor clam Ensis arcuatus (Pharidae: Bivalvia). Aquaculture Research 42: 1549-1557.

Da Costa F, J Aranda-Burgos, A Cerviño-Otero, A Fernandez-Pardo, A Louzán, S Novoa, J Ojea \& D Martinez-Patiño. 2012. Clam hatchery and nursery culture. In: Da Costa F (ed). Clam fisheries and aquaculture, pp. 217-253. Nova Science Publishers, New York.

Da Costa B, J Barreiro, J Ojea, S Novoa, D Martínez-Patiño. 2015. Effects of stocking density on intermediate culture of the razor clam Ensis arcuatus (Pharidae: Bivalvia). Aquaculture Research 46(8): 1858-1865.

Dunham A, H Gurney-Smith, N Plamondon, S Yuan \& CM Pearce. 2013. Aquaculture potential of the basket cockle (Clinocardium nuttallii). Part 1: effects of stocking density on first year grow-out performance in intertidal and off-bottom suspended culture. Aquaculture Research 44: 1236-1253.

Eldridge PJ, AG Eversole \& JM Whetstone. 1979. Comparative survival and growth rates of hard clams, Mercenaria mercenaria, planted in trays subtidally and intertidally at varying densities in a South Carolina estuary. Proceedings of the National Shellfisheries Association 69: 30-39.

Epelbaum A, CM Pearce, N Plamondon, NSYuan \& H Gurney-Smith. 2011. Effects of stocking density and substratum on the survival, growth, burrowing behaviour and shell morphology of juvenile basket cockle, Clinocardium nuttallii: implications for nursery seed production and field outplanting. Aquaculture Research 42: 975-986. 
Hadley N, J Manzi, A Eversole, R Dillon, C Battey \& N Peacock. 1997. A manual for the culture of the hard clam Mercenaria spp in South Carolina, 135 pp. Sea Grant Publications, Charleston.

Jaramillo EH, H Contreras, O Garrido, C Gallardo, J Núñez \& G Jerez. 2008. Estudio de reproducción y crecimiento del recurso Taquilla (Mulinia sp.) en la VIII y X Región. Informe Final Proyecto FIP 2006-51: 1-141. <http://www. subpesca.cl/fipa/613/articles-89168_informe_final.pdf>

Jones GG, CL Sanford \& BL Jones. 1993. Manila clam: Hatchery and nursery methods, $50 \mathrm{pp}$. Innovative Aquaculture Products, Skerry Bay.

Liu W, CM Pearce, AO Alabi, A Beerens \& H Gurney-Smith. 2011. Effects of stocking density, ration, and temperature on growth of early post-settled juveniles of the basket cockle, Clinocardium nuttallii. Aquaculture 320(1-2): 129-136.

Manzi JJ \& M Castagna. 1989. Nursery culture of clams in North America. In: Manzi JJ \& M Castagna (eds). Clam mariculture in North America 5: 127-147. Elsevier, New York.

Manzi, J, N Hadley \& MB Maddox. 1986. Seed clam, Mercenaria mercenaria, culture in an experimental-scale upflow nursery system. Aquaculture 54: 301-311.

Oliva D, A Cifuentes, A Abarca, R Farlora, P Vera, R Durán, J Urra, A Urra, D Brown, A Celis \& R Gutiérrez. 2005. Cultivo comercial de la almeja fina chilena, Mulinia edulis, 60 pp. Universidad de Valparaíso, Valparaíso.

Oliva D, A Abarca, R Gutiérrez, Á Celis, L Herrera \& V Pizarro. 2013. Effect of stocking density and diet on growth and survival of post-larvae of the taquilla clam Mulinia edulis (King \& Broderip, 1832) cultivated in sand. Revista de Biología Marina \& Oceanografía 48(1): 37-44.

Oliva D, A Abarca, R Gutiérrez, L Herrera, A Celis \& LR Durán. 2014. Effect of food ration and stocking density on growth and survival of veliger and pediveliger larvae of the taquilla clam Mulinia edulis reared in the laboratory. Revista de Biología Marina y Oceanografía 49(3): 567-575.

Oostlander PC, J van Houcke, RH Wijffels \& MJ Barbosa. 2020. Microalgae production cost in aquaculture hatcheries. Aquaculture 525, 735310. <https://doi.org/10.1016/j. aquaculture.2020.735310>
Raghavan G \& CP Gopinathan. 2008. Effects of diets, stocking density and environmental factors on growth, survival and metamorphosis of clam, Paphia malabárica (Chemnitz) larvae. Aquaculture Research 39: 928-933.

Royo A, P Ruiz-Azcona \& R Navajas. 2005. Engorde de almeja japonesa Ruditapes philippinarum (Adams \& Reeve, 1850) con anomalías en el crecimiento producidas durante el preengorde. Boletín Instituto Español de Oceanografía 21(1/4): 407-413.

Sokal R \& FJ Rohlf. 2012. Biometry: The principles and practice of statistics in biological research, $937 \mathrm{pp}$. Freeman and Company, New York.

Stotz W, M Valdebenito, M Romero, G Bernal, L Caillaux, J Aburto, H Contreras, D Lancellotti, N Urriola, P Guajardo, S Baro, G Aquea \& C Cerda. 2008. Estudio reproductivo del recurso almeja en la IV Región. Informe Final Proyecto FIP 2006-46: 1-154. <http://www.subpesca. cl/fipa/613/articles-90416_archivo_01.pdf>

Uriarte I. 2008. Estado actual del cultivo de moluscos bivalvos en Chile. En: Lovatelli A, A Farías \& I Uriarte (eds). Estado actual del cultivo y manejo de moluscos bivalvos y su proyección futura: factores que afectan su sustentabilidad en América Latina. Taller Técnico Regional de la FAO, 20-24 de agosto de 2007, Puerto Montt, Chile. FAO Actas de Pesca y Acuicultura 12: 61-75.

Vivanco G, D Oliva \& A Abarca. 2014. Efecto de dietas en base a microalgas tradicionales, nativas y dietas artificiales sobre el crecimiento y supervivencia en larvas de la almeja "taquilla", Mulinia edulis. Revista de Biología Marina \& Oceanografía 49(2): 339-349.

Von Brand E, AAbarca, G Merino \& W Stotz. 2016. Scallop fishery and aquaculture in Chile: A history of developments and declines. In: Shumway SE \& GJ Parsons (eds). Scallops: Biology, ecology, aquaculture, and fisheries, pp 1047-1071. Elsevier, Amsterdam. 